García, D. (2018). El realismo trágico de la corrupción colombiana: Desarrollo, contabilidad, y gestión del riesgo. Contaduría Universidad de Antioquia, 72, 13-27.

Doi: https://doi.org/10.17533/udea.rc.n72a01

\title{
El realismo trágico de la corrupción colombiana: Desarrollo, contabilidad, y gestión del riesgo
}

Danny García Callejas ${ }^{1}$

danny.garcia@udea.edu.co

Universidad de Antioquia

$1 \quad$ Autor para correspondencia. Dirección postal: Calle 67 No. 58-103 Bloque 13 oficina 106. 
El realismo trágico de la corrupción colombiana: Desarrollo, contabilidad, y gestión del riesgo

Resumen: La corrupción es un flagelo que limita el desarrollo del país, debilitando las estructuras sociales y democráticas de la nación. En este contexto, surge la contabilidad como instrumento fundamental que permite visualizar los abusos administrativos, productivos y sociales. A través de la auditoría y el aseguramiento de la información financiera, económica, social, ambientas y la veeduría ciudadana, se presentan oportunidades que tiene la sociedad para garantizar la probidady la transparencia de las cuentas presentadas en el ámbito público y privado. Este artículo reflexiona sobre la corrupción y muestra que la articulación de la sociedad en torno de los procesos contables, los códigos de ética, la planeación y gestión de riesgos puede reducir los niveles y costos del abuso del poder con fines privados.

Palabras clave: Corrupción, contabilidad, auditoría, gestión de riesgos.

The tragic realism of Colombian corruption: development, accounting, and risk management

Abstract: Corruption is a scourge that limits the country's development, weakening the nation's social and democratic structures. Under this context, accounting arises as fundamental instrument allowing to visualize administrative, productive and social abuses. By means of auditing and the assurance of financial, economic, social, and environmental information, as well as citizen observation, opportunities arise for society to guarantee probity and transparency of accounts submitted in the public and private context. This paper reflects on corruption and shows that society's articulation around accounting processes, codes of ethics and risk planning and management can reduce the levels and costs of power abuse with private ends.

Keywords: Corruption, accounting, auditing, risk management.

Le réalisme tragique de la corruption en Colombie: développement, comptabilité et gestion des risques

Résumé: La corruption est un fléau qui empêche le devéloppement du pays, ce qui affaibli les structures sociales et démocratiques de la nation. C'est dans ce cadre qui survient la comptabilité en tant qu'instrument essentiel qui permet de visualiser les abus adminitratifs, productifs et sociaux. Des opportunités voient le jour grâce à l'audit et à l'assurance de l'information financière, économique, sociale, environnementale, et au control des citoyens, dans le cadre du public et du privé. Cet article contient une réflexion sur la corruption et montre que l'articulation de la société autour des processus comptables, les codes d'étique, la planification et la gestion des riques peuvent réduir les niveaux et les coûts de l'abus du pouvoir à des fins privés.

Mots clés: Corruption, comptabilité, audit, gestions des risques.

O Realismo trágico da corrupção colombiana: desenvolvimento, contabilidade e gestão do risco

Resumo: A corrupção é uma tragédia que limita o desenvolvimento do país, debilitando as estruturas sociais e democráticas da nação. Neste contexto, surge a contabilidade como instrumento fundamental que permite visualizar os abusos administrativos, produtivos e sociais. Através da auditoria e a segurança da informação financeira, econômica, social, ambiental e a vedoria cidadã, presentam-se oportunidades que a sociedade tem para garantir a probidade e a transparência das contas apresentadas no âmbito público e privado. Este artigo reflete sobre a corrupção e mostra como a articulação da sociedade em torno dos processos contábeis, os códigos de ética, o planejamento e a gestão do risco pode diminuir os niveis e custos do abuso do poder com fins privados.

Palavras chave: Corrupção, contabilidade, auditoria, gestão do risco. 


\title{
El realismo trágico de la corrupción colombiana: Desarrollo, contabilidad, y gestión del riesgo
}

\author{
Danny García Callejas \\ Doi: https://doi.org/10.17533/udea.rc.n72a01
}

Primera versión recibida en abril de 2018-Versión final aceptada en mayo de 2018

\section{Introducción}

I a corrupción le cuesta a Colombia entre 3\% y 6\% del producto interno Lbruto. Este flagelo está presente en la sociedad, en los ámbitos privados, públicos y del tercer sector. Lo problemático es que limita el desarrollo del país, consolidando prácticas ineficientes y clientelistas que debilitan las estructuras sociales y democráticas de la nación.

El abuso del poder con fines privados, es decir, la corrupción, promueve inequidades e ineficiencias que reducen el crecimiento económico y la inversión. Estas situaciones pueden surgir por decisión de los mismos individuos que dificultan los procesos administrativos, contables y financieros, con el fin cobrar sobornos o hacer favorecimientos. Esto se expone bien en Yan (2012).

La política redunda en permitir o impedir la corrupción, como lo cuenta Klitgaard (1991). Aunque parezca que este flagelo puede aceitar los engranajes del sistema y crear eficiencia, por el contrario, se convierte en arena que interrumpe los procesos productivos, promueve la contaminación y el abuso ambiental y la cohesión social. La contabilidad permite visibilizar estos abusos.

Es más, la auditoría y fiabilidad del registro e interpretación de la información financiera, económica, social y ambiental, junto con la veeduría ciudadana, se convierten en aliados de la probidad y transparencia. La gestión de riesgos resulta en una estrategia que puede reducir la corrupción, partiendo de la información contable, financiera, social y ambiental.

Así, este escrito reflexiona sobre la corrupción y la gestión de riesgos, en las siguientes secciones: la primera, el contexto de la corrupción; la segunda, corrupción, desarrollo y contabilidad; la tercera, el análisis y gestión de riesgos 
García, D. El realismo trágico de la corrupción colombiana: Desarrollo, contabilidad, y gestión del riesgo

como estrategia en favor de la probidad; y, por último, se presentan unas conclusiones.

\section{El contexto de la corrupción colombiana}

El profesor Klitgaard (1991), cuenta sobre las vicisitudes en la lucha contra la corrupción y los dictadores en Guinea Ecuatorial. Sus investigaciones insisten en que la corrupción aumenta con la ineficiencia, la discrecionalidad, los monopolios, los carteles y la falta de aplicación de la ley.

En esta obra del académico estadounidense, queda la sensación de un realismo trágico, al estilo de Yan (2012), en el que los funcionarios chinos, del partido, por supuesto, abusan de su poder para propio beneficio. Las ineficiencias surgen a propósito, para poder imponer su ley, beneficiar a los suyos y encubrir crímenes, como lo narra este escritor en su novela La República del Vino.

Lo que es real es el uso del poder público con fines privados en Colombia. El 28 de diciembre de 1990, el Gobierno expidió la Ley 53 que prohibió el nepotismo en Colombia. En el municipio de El Molino, en la Guajira, los funcionarios de la administración municipal, sin excepción, debieron renunciar pues el parentesco entre ellos era inconfundible.

Este caso, como el de Odebrecht, sin entrar en detalles, ni mencionar el de carteles como el de la hemofilia o el del síndrome de Down, evoca al realismo mágico de Gabriel García Márquez, pero con un sentido aciago. El discurso del nobel, "La soledad de América Latina", da cuenta de nuestra descabellada corrupción y abuso de poder. Cuenta nuestro escritor colombiano que

\footnotetext{
El general Maximiliano Hernández Martínez, el déspota teósofo de El Salvador que hizo exterminar en una matanza bárbara a treinta mil campesinos, había inventado un péndulo para averiguar si los alimentos estaban envenenados, e hizo cubrir con papel rojo el alumbrado público para combatir una epidemia de escarlatina. (García, 1982, p. 7)
}

Según Fedesarrollo y Dejusticia, en Colombia, entre 2009 y 2016, la Fiscalía General de la Nación registró 3.966 casos con al menos una condena por delitos relacionados con formas de corrupción (cohecho por dar u ofrecer, peculado por apropiación y concusión). El soborno, el clientelismo, la extorsión y el nepotismo continúan como formas predominantes de la corrupción en el país.

Estas prácticas nocivas, también, enferman nuestro sistema de salud. iParadójico! El Grupo de Economía de la Salud de la Universidad de Antioquia con su "Estudio de caracterización de riesgos de corrupción y opacidad en el sistema de salud colombiano" a través de una encuesta aplicada a 3.215 personas mayores de 18 años, encuentra una corrupción rampante en el sistema y en el que ningún actor se salva. La mala fama abunda, al igual que la microcorrupción. 
Con tristeza intuíamos esta situación, ahora confirmada. Los tomos de la Corrupción en Colombia, investigación realizada por la Universidad Externado en cuatro tomos, en 2018, da cuenta de una sociedad invadida por actos corruptos. Al parecer, cuentan en los capítulos y tomos, pocos colombianos desconocen $\mathrm{o}$ ha estado alejados de la corruptela nacional. Así, resulta necesario continuar la discusión sobre este tema. La conclusión, no puede ser, que para acabar con la corrupción debemos legalizarla o como diría un conocido político: "llevarla a sus justas proporciones". De esa manera solo resultaría imaginable un realismo trágico.

\section{Corrupción, desarrollo y contabilidad}

La corrupción tiene diversas definiciones que describen atributos como: las asimetrías de poder (Lee-Chai, Chen y Chartrand, 2002);(Johnston, 2005) y la falta de información (Rose-Ackerman, 1999). Bayley (1966) define la corrupción como el mal uso de la autoridad para obtener beneficios personales, incluido cualquier tipo de beneficio personal no monetario (p. 720). Werlin (1973) lo describe como el uso de recursos públicos para actividades privadas. Warren (2004 y 2006) proporciona una explicación detallada sobre la evolución del concepto de corrupción. Transparency International (2009) la define como "el mal uso del poder otorgado para obtener beneficios privados".

Este flagelo puede generar efectos diversos sobre la economía y la sociedad; rampante en Colombia. La economía ha destacado dos consecuencias: uno positivo, al aceitar la maquinaria productiva; y uno negativo, al introducir arena en los engranajes de la economía y disminuir el ritmo de la producción. Las dos subsecciones siguientes dan cuenta de esta discusión, además de detenerse en la importancia de la contabilidad para promover la probidad.

\section{III.1 Corrupción: la grasa que mantiene el sistema funcionando}

Acemoglu y Verdier (1998) sostienen que un cierto nivel de corrupción es óptimo para una economía. Una de las principales funciones del Estado es hacer cumplir los derechos de propiedad. Esto puede ser costoso y conducir a ineficiencias ya que la asignación de estos recursos en otras actividades -educación, salud- puede rendir un mayor rendimiento social. En consecuencia, las economías en desarrollo tienen incentivos para permitir la corrupción debido a su efecto de mejora del crecimiento.

La corrupción surge porque los contratos son incompletos y la presencia de economías informales y subterráneas (Buehn y Schneider, 2012; Dobson y Ramlogan-Dobson, 2012; Dreher y Schneider, 2010). Las relaciones entre los actores privados requieren un tercero que haga cumplir los contratos y promueva la credibilidad y El Estado generalmente toma esta postura. Sin embargo, al hacerlo, los funcionarios del gobierno y las partes interesadas 
privadas pueden tener incentivos para abusar de su poder y obtener ganancias de la ejecución de los contratos.

El enfoque de salarios de eficiencia sugiere que los salarios más altos para los funcionarios del gobierno y los empleados privados previenen la corrupción ya que el costo de ser capturado aumenta.

Sin embargo, utilizar esta estrategia en el sector público atrae a trabajadores calificados y talentosos que abandonan sus empleos privados de baja remuneración en favor del empleo público (Chang y Lai, 2002), creando ineficiencias que llevan a pérdidas de productividad y crecimiento económico más lento (Acemoglu y Verdier, 1998). Una vez más, el sector público tiene incentivos para permitir que cierta corrupción fomente la eficiencia y el crecimiento económico, manteniendo a los trabajadores talentosos en los campos en los que son más productivos.

Acemoglu y Verdier (1998) sugieren que la corrupción es compatible con el crecimiento económico y, por lo tanto, tiene un impacto positivo. Por ejemplo, Bayley (1966) va más allá y afirma que la corrupción es inevitable en los países en desarrollo (p. 719). En consecuencia, Kurer (2001) muestra que la corrupción es abundante en los países desarrollados y endógena como en Italia (Blackburn, Bose y Haque, 2010).

Además, Kurer (2001) sugiere que economías de rápido crecimiento como Tailandia e Italia pueden tolerar la corrupción y aún alcanzar altos niveles de desarrollo. De acuerdo con esto, Meón y Weill (2010) argumentan que la corrupción alienta la eficiencia en países con instituciones débiles, aunque los niveles crecientes de desarrollo reducen la corrupción a través de salarios crecientes que hacen costosa la caza de rentas (Dzhumashev, 2014).

Kurer (2001) explica que existe un umbral para la corrupción que no afecta el crecimiento económico y promueve el desarrollo. Al describir el caso tailandés, Kurer (2001) argumenta que prevalece la corrupción en las zonas rurales del país, que son las más pobres. Sin embargo, dado que las áreas urbanas proporcionan la mayoría de los ingresos fiscales, también demandan más inversiones públicas; dejando menos para las áreas rurales. En consecuencia, la corrupción actúa como un medio de redistribución del ingreso ya que se ponen a disposición más recursos para las áreas rurales, que de otro modo serían retenidos. Dado que las áreas productivas — urbanas - se ven marginalmente afectadas, el crecimiento económico continúa impulsando la demanda al aumentar el ingreso de los ciudadanos rurales.

De manera similar, Leff (1964) y Lui (1985) argumentan que la corrupción estimula el crecimiento al superar la burocracia excesiva y la burocracia gubernamental. En consecuencia, el sector privado toma menos tiempo para poner en marcha un negocio, realizar una exportación o desarrollar empresas conjuntas con empresas multinacionales que atraen inversión y crecimiento 
económico; este es el caso de Perú y Uganda donde los sobornos mejoran el servicio público a costa de eficiencia para la sociedad (Hunt y Laszlo, 2012). Esto explica la corrupción como la grasa que permite que el motor económico funcione sin problemas.

\section{III.2 El costo de la corrupción y la contabilidad}

En contraste con el argumento del dinero, Myrdal (1972) sugiere que la corrupción actúa como un freno para el crecimiento económico ya que los funcionarios públicos tienen incentivos para demorar los procesos burocráticos para obtener sobornos. En otras palabras, los funcionarios públicos crean la ilusión de la burocracia y un complejo sistema burocrático para que puedan aprovecharse de los agentes económicos y hacerles pagar un soborno para acelerar un proceso que han demorado deliberadamente.

La literatura señala tres contraargumentos principales de la corrupción como promotor del crecimiento económico. El primero es el argumento de Myrdal (1972) recién presentado. Los funcionarios públicos, para capturar los recursos privados en su beneficio, crean deliberadamente las regulaciones que ralentizan las empresas económicas; hay evidencia para América Latina a este respecto (Şeker y Yang, 2014). El segundo afirma que la corrupción rara vez crea eficiencia, ya que los buscadores de rentas y las empresas con los productos de menor calidad tienden a sobornar. El tercero, la corrupción crea barreras de entrada, dado que los funcionarios públicos favorecerán a ciertas empresas, los demás quedan en desventaja y, a veces, sin acceso al mercado. En consecuencia, disuade la competencia, la calidad, los bajos precios y la eficiencia (Fisher, 2006, p. 138).

Existe una amplia literatura que respalda el argumento de que la corrupción afecta negativamente el crecimiento económico. Sin embargo, estos estudios no explican si el efecto es a través de las instituciones o si la democracia desempeña un papel importante en este proceso: Colombia es una democracia estable con altos niveles de corrupción que varían dentro del país (Langbein y Sanabria, 2013). Cuando los estudios dan cuenta de estos efectos, generalmente los resultados son inconcluyentes sobre la naturaleza de la relación y, por lo tanto, sobre las posibles recomendaciones de política. Los problemas de endogeneidad son una constante en este tipo de estudios ya que nuestro conocimiento de las causas del crecimiento se atribuye a múltiples factores (Sala-i-Martin, 1997), sin embargo, el tipo, la magnitud y los canales de los efectos de realimentación de las causas de crecimiento están aún por determinar. La Tabla 1 resume artículos que exploran los efectos de la corrupción. 
García, D. El realismo trágico de la corrupción colombiana: Desarrollo, contabilidad, y gestión del riesgo

Tabla 1. Canales y efectos de la corrupción en la economía.

\begin{tabular}{|c|c|}
\hline Artículo & Conclusión principal \\
\hline $\begin{array}{l}\text { Tanzi (1999) y Mironov y Zhuravskaya } \\
\text { (2016) }\end{array}$ & Las organizaciones más ineficientes recurren más a la corrupción. \\
\hline $\begin{array}{l}\text { lindriks, Keen y Muthoo (1999) y } \\
\text { avoodi y Tanzi (1997) }\end{array}$ & \\
\hline $\begin{array}{l}\text { Kaufmann y Wei (1999); Ratbek } \\
\text { Dzhumashev (2014) y Dimant y Tosato } \\
\text { 2018) }\end{array}$ & $\begin{array}{l}\text { La corrupción puede tener un efecto ambiguo sobre el desarrollo, } \\
\text { aunque en general es negativo; el tamaño del gobierno importa. } \\
\text { Sin embargo, la literatura enfatiza en tiene un gran impacto en el } \\
\text { desarrollo económico y social. }\end{array}$ \\
\hline $\begin{array}{l}\text { Mauro (1995); Hines (1995); Galang } \\
\text { (2012) }\end{array}$ & $\begin{array}{l}\text { La corrupción reduce el crecimiento económico, aunque con un } \\
\text { efecto ambiguo sobre el conjunto de la economía. }\end{array}$ \\
\hline $\begin{array}{l}\text { Rose-Ackermann (1975, 1999); } \\
\text { Leite and Weidmann (1999); Collier } \\
\text { y Hoeffler (1998); Collier (2003); } \\
\text { Bhattacharyya y Hodler (2010); Li and } \\
\text { Wu (2010) }\end{array}$ & $\begin{array}{l}\text { Instituciones incluyentes, consolidadas y vigorosas y la confianza } \\
\text { mitigan los efectos negativos de la corrupción y promueven la } \\
\text { probidad. }\end{array}$ \\
\hline Uslaner (2008) & $\begin{array}{l}\text { Las instituciones incluyentes promueven menor corrupción en } \\
\text { regímenes democráticos. }\end{array}$ \\
\hline Ali y Crane (2002) & $\begin{array}{l}\text { La libertad económica reduce la corrupción y sus efectos } \\
\text { negativos sobre la economía. }\end{array}$ \\
\hline $\begin{array}{l}\text { Wei (2001); Mathur y Singh (2013); } \\
\text { O'Toole and Tarp (2014) }\end{array}$ & $\begin{array}{l}\text { Poca evidencia sobre el efecto positivo o de engrase a los } \\
\text { engranajes de la corrupción sobre la inversión. }\end{array}$ \\
\hline
\end{tabular}

Fuente: elaboración propia.

El desafío de establecer una relación sólida entre crecimiento económico y corrupción radica en la dificultad de capturar estos conceptos en un análisis cuantitativo (Sampford, 2006) y la falta de una definición exhaustiva del concepto. Parece que los estudios de corrupción seguirán un camino fútil hasta que su relación con la democracia y las instituciones no esté bien establecida (Warren, 2004). Además, tal vez una perspectiva crítica que incluya las relaciones de poder, la desigualdad y la lucha de clases podría proporcionar una estructura integral sobre la naturaleza de la corrupción (Girling, 1997, p.167) y ofrecer recomendaciones de políticas viables.

No obstante, el estado del arte de Dimant y Tosato (2018) indica que los efectos de la corrupción sobre la economía son grandes y significativos. Las consecuencias son sobre la economía y la sociedad. La microcorrupción termina generando costumbres y hábitos que atenta contra el tejido social y la cohesión ciudadana, debilitando la veeduría ciudadana y participación democrática. En general, la corrupción reduce el crecimiento de la economía, a través de dicha desconfianza y deterioro de las instituciones y la tranquilidad 
de las comunidades. Al igual sucede con los conglomerados empresariales, la corrupción afecta los hábitos y la cultura organizacional.

Luego, erradicar las prácticas organizacionales y de la sociedad que conducen a la corrupción será en vano, de ignorar la forma en que analizamos, registramos e interpretamos la información sobre los hechos económicos, sociales y ambientales. Sin duda, la contabilidad tiene un papel clave en la lucha contra la corrupción. La Tabla 2 comenta sobre el papel de la contabilidad en la disminución de la corrupción, indicando que la fidelidad en los registros, interpretación integral incluyendo códigos de ética y principios morales, al igual que el desarrollo de una auditoría independiente y centrada en las personas para la rendición de cuentas, contribuye a la probidad.

Tabla 2. Corrupción y contabilidad

\begin{tabular}{|c|c|}
\hline Artículo & Papel de la contabilidad \\
\hline Argandoña (2007) & $\begin{array}{l}\text { Fidelidad con la interpretación y registro de los hechos económicos, financieros } \\
\text { y contables. } \\
\text { Defensa de la integridad en el desarrollo de auditorías y supervisión de la } \\
\text { información contable. } \\
\text { Precisar y dar detalle de la información elaborada, creada y analizada, con base } \\
\text { en los registros de la organización. } \\
\text { Coherencia con las normas y estándares oficiales establecidos para el manejo, } \\
\text { tratamiento y análisis de la información. } \\
\text { Establecer criterios para la información dudosa o interpretaciones que generen } \\
\text { riesgos de corrupción o inviten a esta en las decisiones de la organización. } \\
\text { Inclusión de interpretaciones morales y de los códigos de ética de la organización } \\
\text { para promover transparencia y probidad en la toma de decisiones. }\end{array}$ \\
\hline $\begin{array}{l}\text { Malagueño, Albrecht, } \\
\text { Ainge y Stephens } \\
\text { (2010) y Brusca, Rossi } \\
\text { y Aversano (2017) }\end{array}$ & $\begin{array}{l}\text { Auditorías y contabilidad débiles impiden la rendición de cuentas, generando } \\
\text { asignación ineficiente de los recursos. } \\
\text { La contabilidad da transparencia a las transacciones financieras y económicas de } \\
\text { la organización. } \\
\text { Mejores sistemas contables y de auditoría están asociados con menor corrupción. }\end{array}$ \\
\hline $\begin{array}{l}\text { Barkemeyer, Ralf, } \\
\text { Preuss y Lee(2015) }\end{array}$ & $\begin{array}{l}\text { La contabilidad permite que las organizaciones reporten sobre su estado } \\
\text { financiero, económico, social y ambiental; ámbitos que afecta la corrupción. } \\
\text { Los informes de sostenibilidad y la transparencia en la información ayudan en } \\
\text { combatir la corrupción. Un contexto corrupto disminuye la efectividad de estas } \\
\text { herramientas. }\end{array}$ \\
\hline $\begin{array}{l}\text { Houque y Monem } \\
\text { (2016) y Figoli et al. } \\
(2017)\end{array}$ & $\begin{array}{l}\text { La adopción de normas internacionales de información financiera puede reducir } \\
\text { la percepción de corrupción. Su efecto puede ser ambiguo. }\end{array}$ \\
\hline $\begin{array}{l}\text { Jeppesen (2018) } \\
\text { y Gustavson y } \\
\text { Sundström (2016) }\end{array}$ & $\begin{array}{l}\text { La auditoría resulta crucial en el combate de la corrupción, pero debe ser explícita } \\
\text { en incluir controles preventivos y un marco conceptual para poder combatirla. } \\
\text { La auditoría, para ser efectiva en la promoción de la probidad, requiere de: } \\
\text { profesionalismo, independencia y reconocimiento de las personas como los } \\
\text { principales. }\end{array}$ \\
\hline
\end{tabular}

Fuente: elaboración propia. 
García, D. El realismo trágico de la corrupción colombiana: Desarrollo, contabilidad, y gestión del riesgo

La contabilidad cumple un papel central en mitigar y disminuir los efectos y acciones corruptas en la sociedad. La supervisión, auditoría y fidelidad en la gestión, generación e interpretación de información y hechos en las organizaciones y sociedad con base en códigos de ética pueden contribuir al fortalecimiento de la probidad. Sin embargo, los riesgos de la corrupción son latentes y la prevención es un adecuado remedio. La siguiente sección comenta sobre la planeación necesaria para evitar dichos riesgos.

\section{El análisis y gestión de riesgos como estrategia en favor de la probidad}

Según estimaciones de la Contraloría General de la República, Langbein y Sanabria (2013) y Transparencia Internacional, la corrupción en Colombia oscila entre el 3\% y 6\% del producto interno bruto. Este costo considerable llama por la prevención como medida necesaria que permita asignar estos recursos en sectores como salud y educación.

Sin duda, las actividades económicas presentan riesgos de corrupción, pues implican la generación de valor y su realización en el consumo, producción, intercambio y distribución. Las actividades que usan mayores volúmenes de efectivo presentan una mayor probabilidad de que los riesgos se conviertan en hechos (Singh y Bhattacharya, 2017).

En Colombia son recurrentes el favoritismo, clientelismo y tráfico de influencia, con diferencias regionales significativas como lo muestran Langbein y Sanabria (2013). Menores niveles de desarrollo están asociados con instituciones débiles, típico en los municipios más pequeños del país. Esta condición puede derivar en deshonestidad y opacidad en las decisiones económicas y sociales en este nivel, más aún en un contexto de narcotráfico y debilidad democrática (Gómez, 2018).

Allí resulta importante evaluar, desarrollar, implementar y aplicar planes de administración y monitoreo del riesgo resultan para mitigar los efectos, evitar situaciones adversas y promover la probidad. Ello requiere reconocer las acciones, impactos y actores presentes en las instituciones de gobierno. El manejo ordenado y riguroso de la información corporativa e institucional es un insumo fundamental para un plan riguroso e integral (Casadesús de Mingo y Cerrillo-i-Martíneza, 2018).

Un plan de evaluación de riesgo debería contemplar, aunque no limitarse a: seguridad y protección de los ciudadanos; normativa colombiana obligatoria; posibles errores de contratación, trámite y asignación de recursos; políticas públicas sectoriales existentes y futuras; legislación que impacte al área de la actividad económica, social y pública; y externalidades ambientales y sociales producidas por actividades de la institución gubernamental y organizaciones privadas. 
Importante emprender un pacto social. La corrupción es un asunto de la sociedad y los ciudadanos. La rendición de cuentas es central y dichos fenómenos se presentan en los sectores sin distingo. Suponer que es un asunto solo público es un sesgo que impide promover la probidad y la transparencia. La lucha contra la corrupción y mitigación de riesgos es más efectiva en cuanto más sólidas sean las instituciones, las libertades civiles y la democracia (Krishnamurti, Shams y Velayutham, 2018).

Así, la administración del riesgo en los sectores público, privado y tercer sector resulta beneficioso para: controlar el riesgo corporativo, financiero, administrativo y organizacional; dar adecuado uso a la asignación y uso de los recursos, dando cuenta de los procesos para su correcta gestión; fortalecer la participación ciudadana, empresas y organizaciones sociales; facilitar y optimizar la administración de proyectos; manejo de residuos sólidos y efectos ambientales.

Evitar el desarrollo de planes de prevención y de evaluación del riesgo puede tener efectos significativos de largo plazo. Así, podría ignorar la participación de empleados, ciudadanos y comunidad en general en los procesos públicos, en especial, pero también los privados que generan efectos sociales y económicos sin intención. También, podría resultar en pérdidas financieras, económicas, ambientales y sociales que procederían de ineficiencias creadas por el ocultamiento o uso indebido de información.

En general, la evaluación de los riesgos de la corrupción debe ser una prioridad en un país en el que este flagelo es rampante. Para lograr una efectiva evaluación del riesgo debemos procurar al menos cinco pasos: establecer el contexto y características de la comunidad beneficiada y perjudicada; identificar los riesgos por medio de las metas, actividades y estrategias establecidas por la organización; analizar y comprender los riesgos, a la luz de la responsabilidad pública y social, los códigos de ética, los planes establecidos y la rendición de cuentas; y, valorar y evaluar los riesgos con base en la información histórica y experiencias relacionadas en instituciones y organizaciones similares.

$\mathrm{Al}$ evaluar los riesgos se debe tener en cuenta la probabilidad de que suceda y el impacto que tendría para la organización. Allí debemos aceptar el riesgo o gestionarlo; pero identificar los riesgos. Allí tendremos tres alternativas: evitarlo, transferirlo o controlarlo. En todo caso los efectos son para la sociedad por lo que deben ser valorados como tal, aún si los objetivos de la organización son privados.

Entonces, es importante que los planes de administración del riesgo sean revisados incluyendo auditorías, análisis de incidentes, quejas de usuarios, encuestas y reportes del avance de la estrategia para mitigar los efectos esperados - un análisis decidido debe darse frente a los efectos inesperados-. 
García, D. El realismo trágico de la corrupción colombiana: Desarrollo, contabilidad, y gestión del riesgo

El restante, luego de aplicar controles, será el riesgo residual; un reto para una sociedad sumida en la corrupción.

\section{Conclusiones}

La corrupción en Colombia tiene costos considerables. El escenario de arena en el engranaje de la economía es el que aplica para el país, en el entendido que éstas acciones generan ineficiencia y debilitan los arreglos institucionales existentes. De hecho, reduce el desarrollo económico y social, al igual que el crecimiento económico.

Sin embargo, la contabilidad, a través de la auditoría, la generación e interpretación responsable de información financiera, ambiental y social, contribuye a generar probidad en el país. Estas acciones requieren de profesionalismo, independencia y reconocimiento de las personas como los principales, de tal suerte que la rendición de cuentas y veeduría ciudadana puedan ser efectivas.

De igual forma, la gestión del riesgo de la corrupción, a través de planes de administración y evaluación del riesgo permiten prevenir sucesos que atentan contra la transparencia y la probidad. Esto requiere de un pacto social que reconozca que las consecuencias de la corrupción son globales, sus efectos son sociales, y el riesgo residual, en últimas, es asumido por la sociedad.

El realismo trágico de la corrupción en el país se evita a través de la articulación de la sociedad en torno al fortalecimiento de los procesos contables, la aplicación de los códigos de ética y la planeación para la gestión del riesgo de la corrupción. De esta manera, el país puede reducir los niveles y costos del abuso del poder con fines privados.

\section{Referencias Bibliográficas}

Acemoglu, D. y Verdier, T. (1998) Property Rights, Corruption and The Allocation Of Talent: A General Equilibrium Approach. Economic Journal, Vol. 108, pp. 1.381-1.403.

Ali, A. M. y Crain, W. M. (2002) Institutional Distortions, Economic Freedom, and Growth. Cato Journal, Vol. 21, pp. 415-426.

Argandoña, Antonio (2007) La corrupción y las empresas. Occasional Paper OP-07-21, Universidad de Navarra.

Barkemeyer, Ralf, Preuss, L. y Lee, L. (2015) Corporate reporting on corruption: An international comparison. Accounting Forum, Vol. 39, No. 4, pp. 349-365.

Bayley, D. H. (1966) The Effects of Corruption in a Developing Nation. The Western Political Quarterly, 19, 719-732.

Bhattacharyya, S. y R. Hodler (2010) Natural Resources, Democracy and Corruption. European Economic Review, Vol. 54, pp. 608-621.

Blackburn, K; Bose, N. y Haque, M.E. (2010) Endogenous corruption in economic development. Journal of Economic Studies, Vol. 37, pp. 4-25. 
Brusca, I; Rossi, F.M. y Aversano, N. (2017) Accountability and Transparency to Fight against Corruption: An International Comparative Analysis. Journal of Comparative Policy Analysis: Research and Practice, forthcoming.

Buehn, A. y F. Schneider (2012) Corruption and the shadow economy: like oil and vinegar, like water and fire. International Tax and Public Finance, Vol. 19, pp. 172-194.

Casadesús de Mingo, Anahí y Agustí Cerrillo-i-Martíneza (2018) Improving records management to promote transparency and prevent corruption. International Journal of Information Management, Vol. 38, No. 1, pp. 256-261.

Chang, J. y Lai, C. (2002) Is the Efficiency Wage Efficient? The Social Norm and Organizational Corruption. The Scandinavian Journal of Economics, Vol. 1, No. 4, pp. 27-47.

Collier, P. (2003) Natural resources, development and conflict: channels of causation and policy interventions. IMF Working Paper, 28.730.

Collier, P. y Hoeffler, A. (1998) On the Economic Causes of Civil War. Oxford Economic Papers, Vol. 50, pp. 563-573.

Davoodi, H. R. y Tanzi, V. (1997) Corruption, Public Investment, and Growth. IMF Working Papers 97/139.

Dimant, E y Tosato, G (2018) Causes and Effects of Corruption: What has Past Decade's Empirical Research Taught Us? A Survey. Journal of Economic Surveys, Vol. 32, No. 2, pp. 335-356.

Dobson, S. y Ramlogan-Dobson, C. (2012) Inequality, corruption and the informal sector. Economics Letters, Vol. 115, pp. 104-107.

Dreher, A. y Schneider, F. (2010) Corruption and the shadow economy: an empirical analysis. Public Choice, Vol. 144, pp. 215-238.

Dzhumashev, R (2014) Corruption and growth: The role of governance, public spending, and economic development. Economic Modelling, Vol. 37, pp. 202-215.

Galang, R. M. N. (2012) Victim or Victimizer: Firm Responses to Government Corruption. Journal of Management Studies, Vol. 49, pp. 429-462.

García, G. (1982). La soledad de América Latina. Obtenido de https:/www.confiar.coop/ uploads/imagenes/Cuadernillos/Documentos\%20pdf/Gabriel_Garcia_Marquez.pdf

Girling, J. (1997) Corruption, Capitalism and Democracy, New York: Routledge.

Gustavson, M. y Sundström, A. (2016) Organizing the Audit Society: Does Good Auditing Generate Less Public Sector Corruption? Administration \& Society.

Hindriks, J.; Keen, M. y Muthoo, A. (1999) Corruption, extortion and evasion. Journal of Public Economics, Vol. 74, pp. 395-430.

Johnston, M. (2005) Syndromes of Corruption: Wealth, Power, and Democracy. Cambridge: Cambridge University Press.

Kaufmann, D. y Wei, S. J. (1999) Does Grease Money Speed Up the Wheels of Commerce? World Bank Policy Research Working Paper No. 2254.

Jeppesen, K.K. (2018) The role of auditing in the fight against corruption. The British Accounting Review, forthcoming.

Klitgaard, R. (1991). Tropical Gangsters: One Man's Experience With Development And Decadence In Deepest Africa. Basic Books. 
García, D. El realismo trágico de la corrupción colombiana: Desarrollo, contabilidad, y gestión del riesgo

Krishnamurti, C; Shams, S; Velayutham, E (2018) Corporate social responsibility and corruption risk: A global perspective. Journal of Contemporary Accounting \& Economics, Vol. 14, No. 1, pp. 1-21.

Kurer, O. (2001) Why do Voters support Corrupt Politicians en: The Political Economy of Corruption, Arvind K. Jain (ed.). New York: Routledge.

Lee-Chai, A. Y.; Chen, S. y Chartrand, T (2002) From Moses to Marcos: Individual Differences in the Use and Abuse of Power en: The use and abuse of power: multiple perspectives on the causes of corruption; A. Y. Lee-Chai \& J. A. Bargh (eds.), New York: Psychology Press, pp. 57-71.

Leff, N. H. (1964) Economic Development through Bureaucratic Corruption. American Behavioral Scientist, Vol. 8, pp. 8-14.

Lui, F. T. (1985) An Equilibrium Queuing Model of Bribery. Journal of Political Economy, Vol. 93, pp. 760-781.

Malagueño, R; Albrecht, C; Ainge, C; Stephens, N (2010) Accounting and corruption: A cross-country analysis. Journal of Money Laundering Control, Vol. 13, No. 4, pp. 372393.

Mathur, A. y Singh, K. (2013) Foreign direct investment, corruption and democracy. Applied Economics, Vol. 45, pp. 991-1002.

Mauro, P. (1995) Corruption and growth. Quarterly Journal of Economics, Vol. 110, pp. 681-712.

Meón, P. G. y Weill, L (2010) Is Corruption an Efficient Grease? World Development, Vol. 38, pp. 244-259.

Mironov, M. y Zhuravskaya, E. (2016) Corruption in Procurement and the Political Cycle in Tunneling: Evidence from Financial Transactions Data. American Economic Journal: Economic Policy, Vol. 8, No. 2, pp. 287-321.

Myrdal, G. (1972) Asian drama; an inquiry into the poverty of nations, Pantheon Books.

O'Toole, C. M. y Tarp F (2014) Corruption and the efficiency of capital investment in developing countries. Journal of International Development, Vol. 26, pp. 567-597.

Rose-Ackerman, S. (1975) The economics of corruption. Journal of Public Economics, Vol. 4, pp. 187-203.

Rose-Ackerman, S. (1999) Corruption and government: causes, consequences, and reform, Cambridge: Cambridge University Press.

Sala-I-Martin, X. (1997) I Just Ran Two Million Regressions. American Economic Review, Vol. 87, pp. 178-183.

Sampford, C. J. G. (2006) Measuring Corruption, Farnham: Ashgate Publishing, Ltd.

Şeker, M. y Yang, J.S. (2014) Bribery solicitations and firm performance in the Latin America and Caribbean region. Journal of Comparative Economics, Vol. 42, pp. 246-264.

Singh, Kumar, S. y Bhattacharya, K (2017) Does easy availability of cash affect corruption? Evidence from a panel of countries. Economic Systems, Vol. 41, No. 2, pp. 236-247.

Tanzi, V. (1999) Governance, Corruption and Public Finance: An Overview en: Governance, Corruption, and Public Financial Management, Salvatore Schiavo-Campo (ed.) Manila: Asian Development Bank. 
Transparency International (2009) Frequently asked questions about corruption: How Do You Define Corruption? Transparency International.

Uslaner, E. M. (2008) Corruption, Inequality, and the Rule of Law. Cambridge: Cambridge University Press.

Warren, M. E. (2004) What does Corruption mean in a Democracy? American Journal of Political Science, Vol. 48, pp. 28-343.

Warren, M. E. (2006) Political Corruption as Duplicitous Exclusion. PS: Political Science \& Politics, Vol. 34, pp. 803-807.

Wei, S. J. (2001) Does Corruption Relieve Foreign Investors of Burden of Taxes and Capital Controls? en: International Taxation and Multinational Activity, James R. Hines (ed.). Chicago: University of Chicago Press, pp. 73-87.

Werlin, H. H. (1973) The Consequences of Corruption: The Ghanaian Experience. Political Science Quarterly, Vol. 88, pp. 71-85.

Yan, M. (2012). The Republic of Wine. Kailas Editorial S L. 